\title{
Indocyanine green fluorescence-guided pulmonary wedge resection in a child: a case report
}

\author{
CH Fung *, MB, BS, MRCS, CT Lau, MB, BS, FRCS, Kenneth KY Wong, PhD, FRCS
}

Department of Surgery, The University of Hong Kong, Hong Kong

* Corresponding author: fungchiheng@gmail.com

Hong Kong Med J 2020;26:345-7

https://doi.org/10.12809/hkmj198206

\section{Case report}

Tissue diagnosis of pulmonary nodules of an undetermined nature can be achieved with thoracoscopic wedge resection. However, localisation of a lesion during surgery can be technically demanding, especially for small deep-seated lesions. We report our technique of indocyanine green (ICG) fluorescence-guided pulmonary wedge resection in a child.

A 4-year-old boy presented with pyrexia of unknown origin associated with cough for 2 weeks. Extensive septic workup including sputum culture, chest X-ray, nasopharyngeal aspirate and urine culture were unremarkable. Mantoux test was positive. Computed tomographic (CT) scan of the thorax to look for occult chest infection showed features suggestive of pulmonary tuberculosis and a 6-mm nodule over the apical segment of the left lower lobe. He completed a 6-month course of antitubercular medication but reassessment scan after 9 months showed a persistent left lower lobe pulmonary nodule and he was referred to our surgical unit for tissue diagnosis.

The patient underwent CT-guided localisation of the pulmonary nodule under general anaesthesia 1 hour prior to thoracoscopic wedge resection. The pulmonary nodule was identified at the apical segment of the left lower lobe, $1.3 \mathrm{~cm}$ from the pleural surface (Fig 1). An 18-gauge guiding needle was inserted by the radiologist to the lesion under CT guidance. Methylene blue $(0.5 \mathrm{~mL})$ and ICG $(0.5 \mathrm{~mL})$ were injected around the lesion via the guiding needle. A hookwire was also placed for localisation as safety backup and adjunct to ICG. The patient was then transferred back to the operating theatre. The target lesion was identified thoracoscopically with guidance of methylene blue dye and ICG fluorescence (KARL STORZ OPAL1 ${ }^{\circledR}$ ). Wedge resection of the target lesion was performed with Endo GIA ${ }^{\mathrm{TM}}$ Ultra Universal $30-\mathrm{mm}$ staplers (Medtronic). Complete excision was confirmed by absence of fluorescence in the remaining left lower lobe on ICG fluorescence imaging (Fig 2). The use of ICG fluorescence enhanced intra-operative localisation of the small pulmonary nodule and facilitated a minimally invasive operation. The patient made an uneventful recovery and was discharged 2 days after surgery. Histology of the wedge-resected specimen confirmed complete excision and showed granulomatous inflammation with focal necrosis and no evidence of malignancy. The patient had resumed full activity at follow-up 1 week after surgery.

\section{Discussion}

Intra-operative localisation of small pulmonary nodules remains a challenge for prompt and complete resection of lesions. It is recommended that preoperative localisation should be performed prior to minimally invasive resection for pulmonary nodules $<10 \mathrm{~mm}$ in diameter or $>5 \mathrm{~mm}$ from the pleural surface. ${ }^{1}$ Various means of preoperative localisation have been reported in children including micro-coil insertion, methylene blue dye injection, and radiotracer labelling. ${ }^{2}$ Hookwire is reported to be safe and useful for localisation of lung nodules in children as well as other situations such as thoracoscopic resection of deep-seated congenital cystic adenomatoid malformation. ${ }^{2,3}$ Although these methods are considered feasible in children, they have their own limitations and risks, such as local

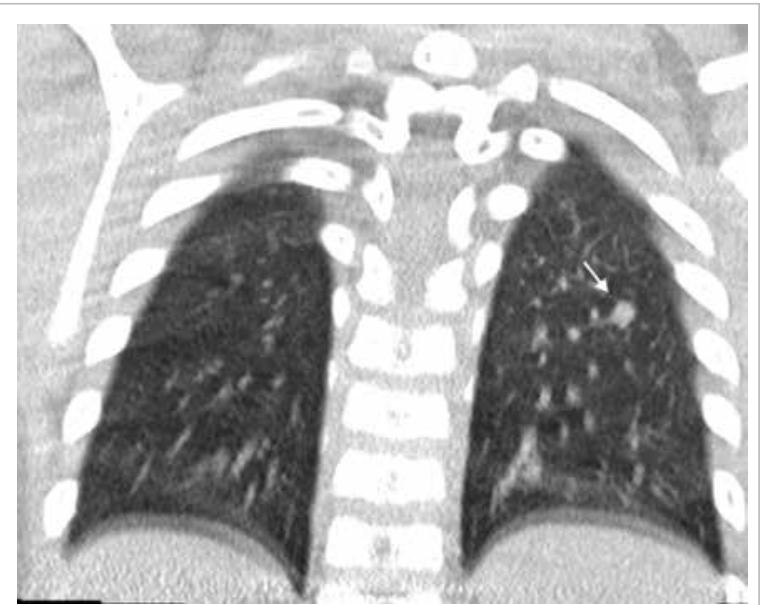

FIG I. Preoperative coronal computed tomography of thorax showing the undetermined lesion (arrow) 


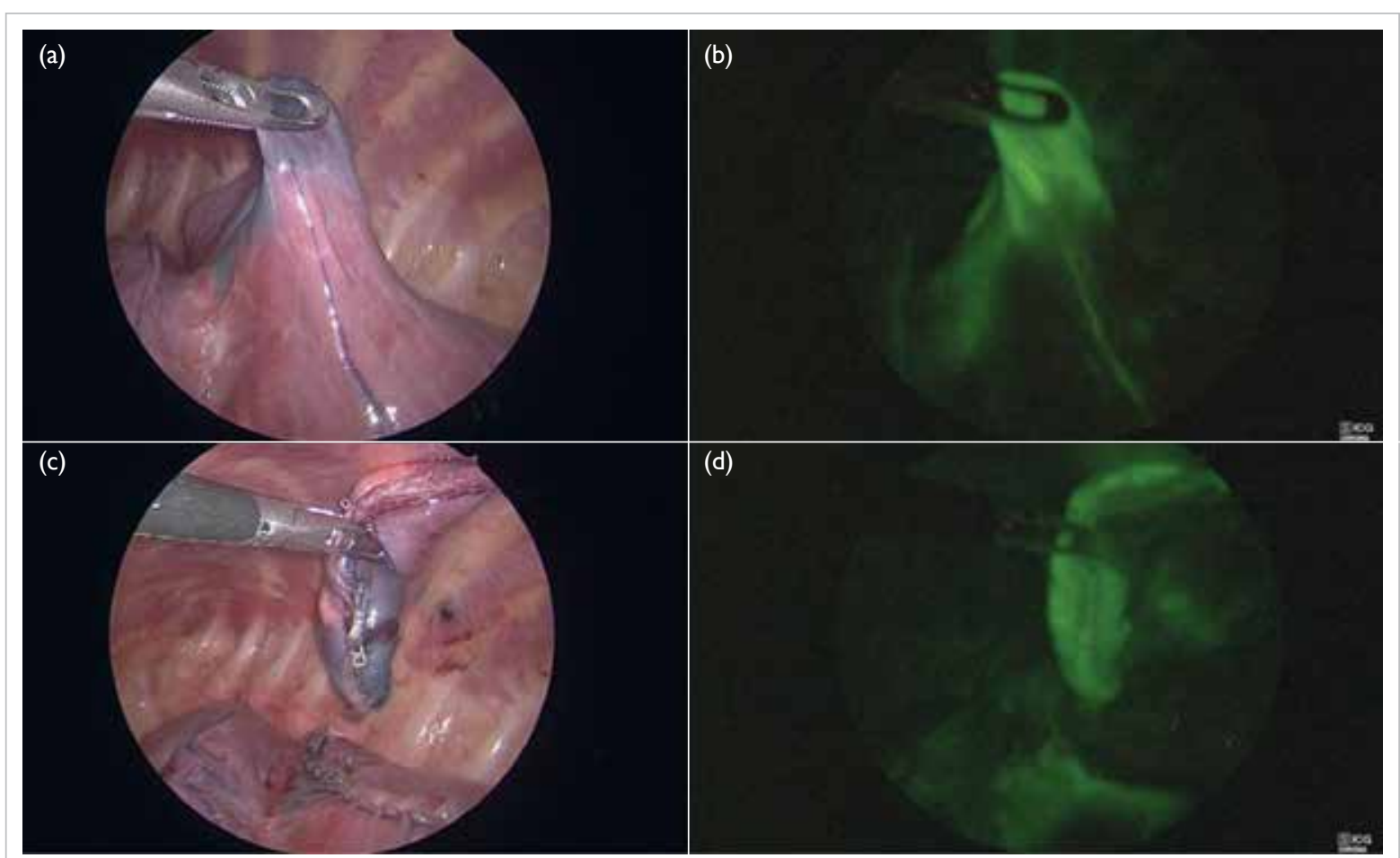

FIG 2. Intra-operative photo showing the segment of lung stained by methylene blue under normal light (a); the same segment of lung seen with indocyanine green fluorescence (b); the wedge of excised lung under normal light (c), and with indocyanine green fluorescence (d), confirming complete resection

trauma and issues of inaccuracy in small lesions for hookwire localisation; difficulty in revealing deep lesions and diffuse spillage in small lesions of methylene blue dye. Efforts have been made to find a convenient, safe, and clear method for localisation.

The United States Food and Drug Administration has recently extended the approved uses of ICG to include sentinel lymph node biopsy in various types of tumour, assessment of blood supply in anastomosis and reconstructive flaps and enhanced visualisation of biliary anatomy during laparoscopic cholecystectomy. ${ }^{4}$ In thoracic surgery, it is a useful aid to sentinel lymph node mapping, lung mapping, oesophageal conduit vascular perfusion, and lung nodule identification. ${ }^{5}$ To date, no data about the use of ICG in thoracoscopic wedge resection in paediatric patients have been reported. In our experience, ICG preoperative localisation is a safe and feasible means to help achieve prompt and complete thoracoscopic wedge resection of small pulmonary nodules. It appeared to be more accurate than hookwire localisation in this patient, allowing immediate clear visualisation when guiding the extent of resection. It also facilitated easy assessment of completeness of resection.

In conclusion, ICG fluorescence is a safe and feasible method of localisation prior to thoracoscopic wedge resection of a pulmonary nodule in children.

\section{Author contributions}

All authors contributed to the concept or design of the study, acquisition of the data, analysis or interpretation of the data, drafting of the manuscript, and critical revision of the manuscript for important intellectual content. All authors had full access to the data, contributed to the study, approved the final version for publication, and take responsibility for its accuracy and integrity.

\section{Conflicts of interest}

As an editor of the journal, KKY Wong was not involved in the peer review process. Other authors have disclosed no conflicts of interest.

\section{Funding/support}

This case report received no specific grant from any funding agency in the public, commercial, or not-for-profit sectors.

\section{Ethics approval}

The patient was treated in accordance with the Declaration of Helsinki. The patient provided written informed consent for all procedures.

\section{References}

1. Suzuki K, Nagai K, Yoshida J, et al. Video-assisted thoracoscopic surgery for small indeterminate pulmonary nodules: indications for preoperative marking. Chest 1999;115:563-8.

2. Polites SF, Fahy AS, Sunnock WA, et al. Use of radiotracer 
labeling of pulmonary nodules to facilitate excisional biopsy and metastasectomy in children with solid tumors. J Pediatr Surg 2018;53:1369-73.

3. Lau CT, Wong KK. Thoracoscopic resection of congenital cystic adenomatoid malformation in a patient with fused lung fissure using hookwire. Innovations (Phila) 2018;13:226-9.
4. Namikawa T, Sato T, Hanazaki K. Recent advances in near-infrared fluorescence-guided imaging surgery using indocyanine green. Surg Today 2015;45:1467-74.

5. Okusanya OT, Hess NR, Luketich JD, Sarkaria IS. Infrared intraoperative fluorescence imaging using indocyanine green in thoracic surgery. Eur J Cardiothorac Surg 2018;53:512-8.

\section{Answers to CME Programme Hong Kong Medical Journal June 2020 issue}

Hong Kong Med J 2020;26:184-91

I. Awareness of fertility preservation among Chinese medical students
A
1. True
2. False
3. False
4. True
5. True
B
1. False
2. False
3. False
4. False
5. True

Hong Kong Med J 2020;26:227-35

II. Hip fractures are preventable: a proposal for osteoporosis screening and fall prevention in older people
A
1. True
2. False
3. True
4. True
5. True
B 1. True
2. False
3. False
4. False
5. True 